\title{
The Inelastic Maxwell Model
}

\author{
E. Ben-Naim ${ }^{1}$ and P. L. Krapivsky ${ }^{2}$ \\ 1 Theoretical Division and Center for Nonlinear Studies, Los Alamos National \\ Laboratory, Los Alamos, NM 87545 \\ 2 Center for Polymer Studies and Department of Physics, Boston University, \\ Boston, MA 02215
}

\begin{abstract}
Dynamics of inelastic gases are studied within the framework of random collision processes. The corresponding Boltzmann equation with uniform collision rates is solved analytically for gases, impurities, and mixtures. Generally, the energy dissipation leads to a significant departure from the elastic case. Specifically, the velocity distributions have overpopulated high energy tails and different velocity components are correlated. In the freely cooling case, the velocity distribution develops an algebraic high-energy tail, with an exponent that depends sensitively on the dimension and the degree of dissipation. Moments of the velocity distribution exhibit multiscaling asymptotic behavior, and the autocorrelation function decays algebraically with time. In the forced case, the steady state velocity distribution decays exponentially at large velocities. An impurity immersed in a uniform inelastic gas may or may not mimic the behavior of the background, and the departure from the background behavior is characterized by a series of phase transitions.
\end{abstract}

\section{Introduction}

Granular gases consist of weakly deformable macroscopic particles that interact via contact interactions and dissipate energy during collisions [1]. In typical experimental situations the system is constantly supplied with energy to counter the loss occurring during inelastic collisions [2]. The most ubiquitous features of granular gases are their tendency to form clusters and their anomalous velocity statistics [3-6]. In turn, these characteristics lead to many interesting collective phenomena including density inhomogeneities [7], shocks $[8,9]$, size segregation [10], pattern formation [11], and phase transitions [12], to name a few.

Inelastic hard spheres provide a useful theoretical, computational, and experimental framework for studying granular gases [13-16]. Inelastic gases pose new theoretical challenges as their dynamics are governed by dissipative collisions and strong velocity correlations. Fundamental equilibrium characteristics such as ergodicity and equipartition of energy are typically violated by this nonequilibrium gas system. As a result, inelastic gases exhibit finite time singularities [17-19], chaotic behavior [20], breakdown of molecular chaos [21-25], and anomalous velocity statistics [26-30].

The significance of the energy dissipation is nicely demonstrated by considering a mean-field version of the hard sphere system, namely a random 
collision process. In this process, randomly chosen pairs of particles undergo inelastic collisions with a random impact direction. This process, often called the Maxwell model, is described by a Boltzmann equation with a velocity independent collision rate. As this model is random in all respects, it is analytically tractable. In fact, in classical kinetic theory of gases, the Maxwell model precedes the Boltzmann equation [31]. Historically, it played an important role in the development of kinetic theory [32-36], and it remains the subject of current research [37-39]. Recently, it has been realized that the Maxwell model is analytically tractable even for inelastic collisions [40-57]. In this review, we detail dynamics of uniform inelastic gases, isolated impurities in uniform gases, and binary mixtures.

First, we consider the one-dimensional gas where explicit analytic solutions are possible (Sec. 2). In the freely cooling case, the velocity distribution approaches a universal (dissipation independent) scaling form with an algebraic high-energy tail. Furthermore, the moments of the velocity distribution exhibit a multiscaling asymptotic behavior, and the velocity autocorrelation function decays algebraically with a non-universal exponent. In the uniformly heated case, the system approaches a steady state, and the velocity distribution has an exponential high-energy tail.

Many of these features extend to higher dimensions (Sec. 3). In the freely cooling case, however, the scaling function underlying the velocity distribution is no longer universal - its shape and its extremal behavior depend on the spatial dimension and the degree of dissipation. We obtain explicit expressions for low order moments and show the development of correlations between different velocity components. Such correlations are more pronounced in the freely cooling case, compared with the forced case.

When an impurity is immersed in a homogeneous cooling fluid, various scenarios are possible (Sec. 4). If the impurity mass is lighter than a critical mass, its velocity statistics are completely governed by the background. As the impurity mass increases, impurity to fluid moment ratios of sufficiently high order diverge asymptotically. A series of critical masses govern these phase transitions. Ultimately, the temperature ratio may diverge, and the impurity becomes so energetic that it effectively decouples from the fluid. In this case, its velocity distribution is distinct from the fluid. Although singletime velocity statistics of the impurity may be governed by the fluid, two-time statistics such as the autocorrelation function are always different.

For mixtures (Sec. 5), all components have the same asymptotic temperature decay, and a high-energy tail as in the uniform gas case. This behavior is shown in detail for one-dimensional freely cooling binary mixtures.

We finally describe a lattice generalization of the Maxwell model where particles are placed on a lattice and only nearest neighbors interact via inelastic collisions (Sec. 6). We show the development of spatial velocity correlations with a diffusively growing correlation length. These correlations significantly reduce the temperature cooling rate. 


\section{Uniform Gases: One dimension}

\subsection{The freely cooling case}

Consider an infinite system of identical particles that interact via instantaneous binary collisions. When a pair of particles with velocities $\left(u_{1}, u_{2}\right)$ collide, their post collision velocities $\left(v_{1}, v_{2}\right)$ are given by the collision rule

$$
v_{1,2}=u_{1,2} \mp(1-p) g,
$$

with $g=u_{1}-u_{2}$ the relative velocity, and $p$ the dissipation parameter. The total momentum is always conserved, $u_{1}+u_{2}=v_{1}+v_{2}$. Since the system is invariant under the Galilean transformation $v \rightarrow v-v_{0}$, we set the average velocity to zero, without loss of generality. In each collision, the relative velocity between the colliding particles is reduced by the restitution coefficient $r=1-2 p$. In an inelastic collision, there is an energy loss, $\Delta E=-p(1-p) g^{2}$ (particle mass is set to unity); energy loss is maximal for completely inelastic collisions $(p=1 / 2)$, and it vanishes for elastic collisions $(p=0)$.

In a random collision process, the collision rate is independent of the particles' velocities. Let $P(v, t)$ be the normalized velocity distribution at time $t$. It evolves according to the Boltzmann equation

$$
\begin{aligned}
\frac{\partial P(v, t)}{\partial t}= & K \int d u_{1} P\left(u_{1}, t\right) \int d u_{2} P\left(u_{2}, t\right) \\
& \times\left\{\delta\left[v-u_{1}+(1-p) g\right]-\delta\left(v-u_{2}\right)\right\} .
\end{aligned}
$$

This Boltzmann equation, with a velocity independent collision kernel, is termed the Maxwell model in kinetic theory [35]. The overall collision rate $K=\sqrt{T}$, with the granular temperature $T=\int d v v^{2} P(v, t)$, is chosen to represent the typical particle velocity. The quadratic integrand in Eq. (2) reflects the random and binary nature of the collision process, while the gain and loss terms reflect the collision rules (1). One can verify that the total number of particles, $\int d v P(v, t)$, and the total momentum, $\int d v v P(v, t)$, are conserved; hereinafter we set $\int d v P(v, t)=1$ and $\int d v v P(v, t)=0$.

For inelastic hard spheres, where the collision rate equals the relative velocity, the equation governing the temperature is part of an infinite hierarchy of equations. In contrast, in the Maxwell model, the temperature obeys a closed equation

$$
\frac{d T}{d t}=-\lambda T^{3 / 2} \quad \text { with } \quad \lambda=2 p(1-p) .
$$

The temperature decays algebraically with time according to Haff's law [58]

$$
T(t)=\frac{T_{0}}{\left(1+t / t_{0}\right)^{2}},
$$


with the time scale $t_{0}^{-1}=p(1-p) \sqrt{T_{0}}$. Eventually, all particles acquire the average velocity: $P(v, \infty)=\delta(v)$. Our goal is to characterize how the velocity distribution approaches this terminal state asymptotically. Since the overall collision rate is uniform, it is useful to characterize time by the collision counter $\tau=\int_{0}^{t} d t^{\prime} K\left(t^{\prime}\right)$, equal to the average number of collisions experienced by a particle,

$$
\tau=\frac{2}{\lambda} \ln \left(1+t / t_{0}\right)
$$

In terms of the number of collisions, $T(\tau)=T_{0} e^{-\lambda \tau}$.

The convolution structure of the Boltzmann equation suggests to apply the Fourier transform $F(k, \tau)=\int d v e^{i k v} P(v, \tau)$. This quantity evolves according to [40]

$$
\frac{\partial}{\partial \tau} F(k, \tau)+F(k, \tau)=F(k-p k, \tau) F(p k, \tau) .
$$

This closed equation is both nonlinear and nonlocal, yet it is analytically tractable. Assuming that the velocity distribution approaches its final state in a self-similar fashion, we seek a scaling solution

$$
P(v, t)=T^{-1 / 2} \mathcal{P}(w) \quad \text { with } \quad w=v T^{-1 / 2} .
$$

The scaling form corresponding to the Fourier transform is $F(k, \tau)=f(z)$ with the variable $z=|k| T^{1 / 2}$. Substituting the scaling ansatz into Eq. (6) and using $d T / d \tau=-\lambda T$, the Fourier scaling function satisfies

$$
-p(1-p) z f^{\prime}(z)+f(z)=f(z-p z) f(p z) .
$$

This equation is supplemented by the small- $z$ behavior $f(z) \cong 1-\frac{1}{2} z^{2}$, which is dictated by the small wave number behavior, $F(k) \cong 1-\frac{1}{2} k^{2} T$. Subject to these conditions, the (unique) solution is [43]

$$
f(z)=(1+z) e^{-z} .
$$

The scaled velocity distribution is obtained by performing an inverse Fourier transform

$$
\mathcal{P}(w)=\frac{2}{\pi} \frac{1}{\left(1+w^{2}\right)^{2}} .
$$

Remarkably, the scaled velocity distribution is independent of the dissipation parameter $p$. Another important feature is the algebraic decay: $\mathcal{P}(w) \sim$ $w^{-4}$ for $w \gg 1$. This behavior should be compared with the exponential high energy tails obtained for the traditional Boltzmann equation [26, 28]. The enhancement in the likelihood of finding energetic particles is due to the effective reduction in their collision rate.

Typically, in kinetic theory of molecular gases, the velocity distributions have sharp tails such that all moments of the distribution are finite ${ }^{1}$. Sonine

\footnotetext{
${ }^{1}$ Algebraic high-energy tails may also characterize nonequilibrium states of elastic gases; for uniform shear flows of two-dimensional Maxwell molecules, this has been recently shown by Acedo, Santos, and Bobylev [38].
} 
expansions, where Maxwellian distributions are systematically modified by polynomials of increasing orders, are widely used to analyze the Boltzmann equation. The Maxwell model can be conveniently utilized to examine the applicability of this approach to situations where the velocity distributions have overpopulated high energy tails.

The scaling function does not characterize all features of the asymptotic time dependent behavior. Higher than third moments of the scaling function diverge, and since moments must be finite at all times, a direct calculation is necessary. Moments of the velocity distribution, $M_{n}(t)=\int d v v^{n} P(v, t)$, obey a closed set of equations

$$
\frac{d}{d \tau} M_{n}+a_{n} M_{n}=\sum_{m=2}^{n-2}\left(\begin{array}{c}
n \\
m
\end{array}\right) p^{m}(1-p)^{n-m} M_{m} M_{n-m}
$$

with $a_{n}(p)=1-p^{n}-(1-p)^{n}$. These equations are solved recursively using $M_{0}=1$ and $M_{1}=0$. Assuming that all moments are initially finite we find that to leading order, the moments asymptotically decay as

$$
M_{n} \sim e^{-a_{n} \tau} \sim t^{-2 a_{n} / a_{2}} .
$$

Indeed, the sum term in (11) is asymptotically negligible because the coefficients satisfy the inequality $a_{n}<a_{m}+a_{n-m}$ for all $1<m<n-1$ [40]. Asymptotically, $M_{n} \sim e^{-a_{n} \tau}$ and $M_{2} \sim e^{-a_{2} \tau}$, so $M_{n} \sim M_{2}^{\alpha_{n}}$ with $\alpha_{n}=a_{n} / a_{2}$. In this so-called multiscaling asymptotic behavior, the temperature does not characterize higher order moments (for ordinary scaling behavior $\alpha_{n}=n / 2$ ). The indices $\alpha_{n}$ increase monotonically with $p$, so the stronger the dissipation, the more pronounced the multiscaling asymptotic behavior.

The scaling behavior characterizes only velocities of the scale of the typical velocity. For sufficiently large velocities, far outside the scaling region, the gain term in the Boltzmann equation is negligible, and $\left(\frac{\partial}{\partial \tau}+1\right) P(v, \tau)=0$. Consequently, a generic exponential decay, $P(v, \tau) \sim P_{0}(v) \exp (-\tau)$ characterizes such large velocities. Similarly, sufficiently large moments decay according to $M_{n} \sim e^{-\tau} \sim t^{-1 /[p(1-p)]}$ for $n \rightarrow \infty$.

In several studies, inelasticity is treated as a small perturbation $[29,30$, 59]. To first order in $p$, Eq. (6) reads $\left(\frac{\partial}{\partial \tau}+p k \frac{\partial}{\partial k}\right) F(k, \tau)=0$. The solution to this equation, $F(k, \tau)=F_{0}\left(k e^{-p \tau}\right)$, remembers the initial conditions forever, in contradiction with the exact asymptotic behavior (10). This example raises questions concerning the validity of perturbation analysis in the vicinity of $p=0$. The $p \rightarrow 0$ limit is singular and indeed, in Eq. (8) the small parameter $p$ multiplies the highest derivative. The singular nature of the quasi-elastic limit has profound general consequences.

The Fourier transform can be expressed as a series expansion. The linear term can be eliminated from Eq. (6) by making the transformation $F \rightarrow e^{\tau} F$ 
and $\tau \rightarrow 1-e^{-\tau}$. Then a formal Taylor expansion solution can be found [42]:

$$
F(k, \tau)=e^{-\tau} \sum_{n=0}^{\infty} \frac{\left(1-e^{-\tau}\right)^{n}}{n !} F_{n}(k) .
$$

The expansion functions $F_{n}(k)$ are obtained recursively: $F_{0}(k) \equiv F(k, \tau=0)$, and generally $F_{n+1}(k)=\sum_{m=0}^{n}\left(\begin{array}{c}n \\ m\end{array}\right) F_{m}(k-p k) F_{n-m}(p k)$. For instance, when the initial distribution is Maxwellian, the expansion functions, and hence the velocity distribution itself consist of sums of Maxwellians. Similarly, starting from a stretched exponential, all expansion functions consist of sums of stretched exponentials. In general, the expansion functions are products of $F_{0}$ 's with stretched arguments. This implies that starting from a compact initial distribution $P(v, 0)$, the velocity distribution $P(v, t)$ develops a set of singularities. For instance, a distribution with support in $\left[-v_{0}, v_{0}\right]$ becomes non-analytic at an infinite set of points $v_{l, m}= \pm p^{l}(1-p)^{m} v_{0}$.

Thus far, we characterized velocity statistics at a specific point in time. The autocorrelation function

$$
A\left(t^{\prime}, t\right)=\overline{v\left(t^{\prime}\right) v(t)}
$$

with the overline denoting an average over all particles quantifies (two-point) temporal correlations in the velocity of a tagged particle. We have for $\tau>\tau^{\prime}$

$$
\begin{aligned}
\frac{d}{d \tau} A\left(\tau^{\prime}, \tau\right) & =\frac{d}{d \tau} \overline{v\left(\tau^{\prime}\right) v(\tau)}=\overline{v\left(\tau^{\prime}\right)[d v(\tau) / d \tau]} \\
& =-(1-p) \overline{v\left(\tau^{\prime}\right)[v(\tau)-u(\tau)]}=-(1-p) A\left(\tau^{\prime}, \tau\right) .
\end{aligned}
$$

This derivation reflects averaging over all possible collisions between the tagged particle of velocity $v$ and another particle of particle of velocity $u$, with the collision rule, $v \rightarrow v-(1-p)(v-u)$. Therefore, the autocorrelation, $A\left(\tau^{\prime}, \tau\right)=A\left(\tau^{\prime}, \tau^{\prime}\right) \exp \left[-(1-p)\left(\tau-\tau^{\prime}\right)\right]$, decays exponentially with the collision number, for $\tau>\tau^{\prime}$ [45]. In terms of the original time variable:

$$
A\left(t^{\prime}, t\right)=A_{0}\left(1+t^{\prime} / t_{0}\right)^{1 / p-2}\left(1+t / t_{0}\right)^{-1 / p},
$$

with $A_{0}=T_{0}$. In particular, $A(t) \equiv A(0, t) \propto t^{-1 / p}$, so memory of the initial conditions decays algebraically with time. The autocorrelation function decays faster than the temperature, $A(t) \leq T(t)$ (the two functions coincide in the completely inelastic case, $p=1 / 2$ ). Generally, the autocorrelation is a function of the waiting time $t^{\prime}$ and the observation time $t$, and not simply of their difference, $t-t^{\prime}$. This history dependence ("aging") merely reflects the fact that the collision rate keeps changing with time.

The spread in the position of a tagged particle, $\Delta^{2}(t) \equiv\left\langle|x(t)-x(0)|^{2}\right\rangle$, is obtained from the autocorrelation function using $\Delta^{2}=2 \int_{0}^{t} d t^{\prime} \int_{0}^{t^{\prime}} d t^{\prime \prime} A\left(t^{\prime \prime}, t^{\prime}\right)$. Substituting the autocorrelation function (16), we find that asymptotically, the spread grows logarithmically with time

$$
\Delta \propto \sqrt{\tau} \propto \sqrt{\ln t} .
$$


This behavior reflects the $t^{-1}$ decay of the overall velocity scale and is consistent with inelastic hard spheres results [26,60-62].

\subsection{The forced case}

In experimental situations, granular ensembles are constantly supplied with energy, typically through the boundaries, to counter the dissipation occurring during collisions ${ }^{2}$. Theoretically, it is convenient to consider uniformly heated systems, as discussed by Williams and MacKintosh [14] in the case of onedimensional inelastic hard rods and then extended to higher dimensions (see [28-30] and references therein). We therefore study a system where in addition to changes due to collisions, velocities also change due to an external forcing: $\left.\frac{d v_{j}}{d t}\right|_{\text {heat }}=\xi_{j}$. We assume standard uncorrelated white noise: $\left\langle\xi_{j}\right\rangle=0$ and $\left\langle\xi_{i}(t) \xi_{j}\left(t^{\prime}\right)\right\rangle=2 \bar{D} \delta_{i j} \delta\left(t-t^{\prime}\right)$.

Such white noise forcing amounts to diffusion with a 'diffusion' coefficient $\bar{D}$ in velocity space. Therefore, the Boltzmann equation (2) is augmented by a diffusion term

$$
\begin{aligned}
\left(\frac{\partial}{\partial t}-\bar{D} \frac{\partial^{2}}{\partial v^{2}}\right) P(v, t)= & K \int d u_{1} P\left(u_{1}, t\right) \int d u_{2} P\left(u_{2}, t\right) \\
& \times\left\{\delta\left[v-u_{1}+(1-p) g\right]-\delta\left(v-u_{2}\right)\right\}
\end{aligned}
$$

The temperature changes according to $d T / d t+\lambda T^{3 / 2}=2 \bar{D}$, so the steady state temperature is $T_{\infty}=(2 \bar{D} / \lambda)^{2 / 3}$. The relaxation toward the steady state is exponential, $\left|T-T_{\infty}\right| \sim \exp (-$ const. $\times t)$.

At the steady state, the Fourier transform $F_{\infty}(k) \equiv F(k, \infty)$ satisfies

$$
\left(1+D k^{2}\right) F_{\infty}(k)=F_{\infty}(k-p k) F_{\infty}(p k) .
$$

with $D=\bar{D} / \sqrt{T_{\infty}}$. Conservation of the total number of particles and the total momentum impose $F_{\infty}(0)=1$ and $F_{\infty}^{\prime}(0)=0$, respectively. The solution is found recursively to give the following infinite product [40]

$$
F_{\infty}(k)=\prod_{l=0}^{\infty} \prod_{m=0}^{l}\left[1+p^{2 m}(1-p)^{2(l-m)} D k^{2}\right]^{-\left(\begin{array}{c}
l \\
m
\end{array}\right)}
$$

Thus in one dimension the Fourier transform is determined analytically in the steady state. To extract the high-energy tail from $(20)$ we note that $F_{\infty}(k)$ has an infinite series of poles located at $\pm i\left[p^{2 m}(1-p)^{2(l-m)} D\right]^{-1 / 2}$. The simple poles at $\pm i / \sqrt{D}$ closest to the origin imply an exponential decay of the velocity distribution $[49,53]$,

$$
P_{\infty}(v) \simeq \frac{A(p)}{v_{*}} e^{-|v| / v_{*}}, \quad \text { with } \quad v_{*}=\sqrt{D}
$$

\footnotetext{
${ }^{2}$ When the mean-free path is comparable with the system size, the boundary effectively plays the role of a thermal heat bath.
} 
when $|v| \rightarrow \infty$. A (lengthy) re-summation similar to that used in [40] yields the residue to this pole, and in turn, the prefactor

$$
\begin{aligned}
A(p) & =\frac{1}{2} \exp \left(\sum_{n=1}^{\infty} \frac{1}{n} \frac{1-a_{2 n}(p)}{a_{2 n}(p)}\right), \\
& =\frac{1}{2} \exp \left(\sum_{n=1}^{\infty} \frac{1}{n} \frac{p^{2 n}+(1-p)^{2 n}}{1-p^{2 n}-(1-p)^{2 n}}\right) .
\end{aligned}
$$

In the interesting quasi-elastic limit $(p \rightarrow 0)$ we expand the denominator. Keeping only the dominant terms simplifies the sum to $\sum_{n=1}^{\infty} \frac{1}{2 p n^{2}}=\frac{\pi^{2}}{12 p}$ and to leading order, the prefactor is $A(p) \propto \exp \left[\pi^{2} /(12 p)\right]$.

A detailed analysis of the next poles shows that for sufficiently small velocities, $v \ll v_{c}$, the velocity distribution is essentially Maxwellian, $\exp \left(-v^{2}\right)$ [57]. For convenience, the velocities are normalized here by the rms velocity. Matching this with the exponential tail $\exp (-|v| / \sqrt{p})$ yields the crossover velocity $v_{c} \sim p^{-1 / 2}$ (this estimate holds up to a logarithmic correction [57]). Thus, although in general the asymptotic decay of the velocity distribution is exponential, in the quasi-elastic limit, the velocity distribution is Maxwellian over a growing velocity range. A similar interplay between a generic $\exp \left(-|v|^{3 / 2}\right)$ tail and a $\exp \left(-|v|^{3}\right)$ decay in the quasi-elastic limit is found for inelastic hards spheres as well [30].

The leading high-energy behavior can also be derived by using a useful heuristic argument $[26,28,49]$. For sufficiently large velocities, the gain term in the collision integral in Eq. (18) is negligible. The resulting equation for the steady state distribution

$$
D \frac{d^{2}}{d v^{2}} P_{\infty}(v)=-P_{\infty}(v)
$$

yields the exponential high-energy tail (21). This argument applies to arbitrary collision rates. For example, if $K \propto v^{\delta}$, the right-hand side in (23) becomes $-v^{\delta} P_{\infty}$ implying that $P_{\infty}(v) \propto \exp \left(-|v|^{\gamma}\right)$ with $\gamma=1+\delta / 2$. For hard spheres $(\delta=1)$ one finds $\gamma=3 / 2[28]$, and curiously, the Gaussian tail arises only for the so-called very hard spheres $(\delta=2)$ [35].

Finally, we notice that steady state properties in the heated case are intimately related with the relaxation properties in the cooling case. This can be seen via the cumulant expansion

$$
F_{\infty}(k)=\exp \left[\sum_{n=1}^{\infty} \psi_{n}\left(-D k^{2}\right)^{n}\right] .
$$

Replacing the term $\left(1+D k^{2}\right)$ with $\exp \left[-\sum_{n=1} n^{-1}\left(-D k^{2}\right)^{n}\right]$, and substituting the cumulant expansion into Eq. (19) yields $\psi_{n}=\left[n a_{2 n}(p)\right]^{-1}$. The cumulants $\kappa_{n}$ are defined via $\ln F_{\infty}(k)=\sum_{n=1} \kappa_{n}(i k)^{n} / n$ !. Therefore, the steady-state cumulants are directly related to the relaxation coefficients (12), $\kappa_{2 n}=\frac{(2 n) !}{n} D^{n} / a_{2 n}$ (the odd cumulants vanish). 


\section{Uniform gases: Arbitrary dimension}

\subsection{The freely cooling case}

In general dimension, the colliding particles exchange momentum only along the impact direction. The post-collision velocities $\boldsymbol{v}_{1,2}$ are given by a linear combination of the pre-collision velocities $\boldsymbol{u}_{1,2}$,

$$
\boldsymbol{v}_{1,2}=\boldsymbol{u}_{1,2} \mp(1-p)(\boldsymbol{g} \cdot \boldsymbol{n}) \boldsymbol{n} .
$$

Here $\boldsymbol{g}=\boldsymbol{u}_{1}-\boldsymbol{u}_{2}$ is the relative velocity and $\boldsymbol{n}$ the unit vector connecting the particles' centers. The normal component of the relative velocity is reduced by the restitution coefficient $r=1-2 p$, and the energy dissipation is given by $\Delta E=-p(1-p)(\boldsymbol{g} \cdot \boldsymbol{n})^{2}$.

In random collision processes, both the impact direction and the identity of the colliding particles are chosen randomly. In such a process, the Boltzmann equation

$$
\begin{aligned}
\frac{\partial P(\boldsymbol{v}, t)}{\partial t}= & K \int d \boldsymbol{n} \int d \boldsymbol{u}_{1} P\left(\boldsymbol{u}_{1}, t\right) \int d \boldsymbol{u}_{2} P\left(\boldsymbol{u}_{2}, t\right) \\
& \times\left\{\delta\left[\boldsymbol{v}-\boldsymbol{u}_{1}+(1-p)(\boldsymbol{g} \cdot \boldsymbol{n}) \boldsymbol{n}\right]-\delta\left(\boldsymbol{v}-\boldsymbol{u}_{1}\right)\right\}
\end{aligned}
$$

exactly describes the evolution of the velocity distribution function $P(\boldsymbol{v}, t)$. The overall collision rate is chosen to represent the typical relative velocity, $K=\sqrt{T}$, with the granular temperature now being the average velocity fluctuation per degree of freedom, $T=\frac{1}{d} \int d \boldsymbol{v} v^{2} P(\boldsymbol{v}, t)$ with $v \equiv|\boldsymbol{v}|$. The evolution equation involves integration over all impact directions, and this angular integration should be normalized, $\int d \boldsymbol{n}=1$. We tacitly ignored the restriction $\boldsymbol{g} \cdot \boldsymbol{n}>0$ on the angular integration range in Eq. (26) since the integrand obeys the reflection symmetry $\boldsymbol{n} \rightarrow-\boldsymbol{n}$.

Several temporal characteristics such as the temperature and the autocorrelation behave as in the one-dimensional case. For example, the temperature satisfies Eq. (3) with prefactor $\lambda=2 p(1-p) / d$ reduced $^{3}$ by a factor $d$. The temperature therefore decays according to Haff's law (4), with the time scale $t_{0}=d /\left[p(1-p) \sqrt{T_{0}}\right]$ set by the initial temperature. Similarly, the decay rate of the autocorrelation function is merely reduced by the spatial dimension, $\frac{d}{d \tau} A\left(\tau^{\prime}, \tau\right)=-\frac{1-p}{d} A\left(\tau^{\prime}, \tau\right)$. Consequently, the nonuniversal decay (16) and the logarithmic spread (17) hold in general.

The Fourier transform, $F(\boldsymbol{k}, t)=\int d \boldsymbol{v} e^{i \boldsymbol{k} \cdot \boldsymbol{v}} P(\boldsymbol{v}, t)$, satisfies

$$
\frac{\partial}{\partial \tau} F(\boldsymbol{k}, \tau)+F(\boldsymbol{k}, \tau)=\int d \boldsymbol{n} F(\boldsymbol{k}-\boldsymbol{p}, \tau) F(\boldsymbol{p}, \tau)
$$

\footnotetext{
${ }^{3}$ The reduction in rate by a factor $d$ is intuitive because of the $d$ independent directions only the impact direction is relevant in collisions. Mathematically, the prefactor $\lambda=2 p(1-p) \int d \boldsymbol{n} n_{1}^{2}$ is computed by using the identity $n_{1}^{2}+\ldots+n_{d}^{2}=1$ that yields $\int d \boldsymbol{n} n_{1}^{2}=1 / d$.
} 
with $\boldsymbol{p}=(1-p)(\boldsymbol{k} \cdot \boldsymbol{n}) \boldsymbol{n}$ reflecting the momentum transfer occurring during collisions. This equation was obtained by multiplying Eq. (26) by $e^{i \boldsymbol{k} \cdot \boldsymbol{v}}$ and integrating over the velocities. The power of the Fourier transform is even more remarkable in higher dimensions ${ }^{4}$ as it reduces the $(3 d-1)$-fold integral in Eq. (26) to the $(d-1)$-fold integral in Eq. (27).

Hereinafter, we consider only isotropic velocity distributions. The Fourier transform depends only on $k \equiv|\boldsymbol{k}|$, so we write $F(\boldsymbol{k}, \tau)=F(y, \tau)$ with $y=$ $k^{2}$. To perform the angular integration, we employ spherical coordinates with the polar axis parallel to $\boldsymbol{k}$, so that $\hat{\boldsymbol{k}} \cdot \boldsymbol{n}=\cos \theta$. The $\theta$-dependent factor of the angular integration measure $d \boldsymbol{n}$ is proportional to $(\sin \theta)^{d-2} d \theta$. Denoting angular integration with brackets, $\langle f\rangle \equiv \int d \boldsymbol{n} f$, and using $\mu=\cos ^{2} \theta$ gives

$$
\langle f\rangle=\int_{0}^{1} d \mu \frac{\mu^{-\frac{1}{2}}(1-\mu)^{\frac{d-3}{2}}}{B\left(\frac{1}{2}, \frac{d-1}{2}\right)} f(\mu),
$$

where $B(a, b)$ is the beta function. This integration is properly normalized, $\langle 1\rangle=1$. The governing equation (27) for the Fourier transform can now be rewritten in the compact from

$$
\frac{\partial}{\partial \tau} F(y, \tau)+F(y, \tau)=\langle F(\xi y, \tau) F(\eta y, \tau)\rangle
$$

with the shorthand notations $\xi=1-\left(1-p^{2}\right) \mu$ and $\eta=(1-p)^{2} \mu$. Unlike the one-dimensional case, explicit solutions of this nonlinear and nonlocal rate equation are cumbersome and practically useless. Nevertheless, most of the physically relevant features of the velocity distributions including the large velocity statistics and the time dependent behavior of the moments can be obtained analytically.

We seek a scaling solution: $P(\boldsymbol{v}, t)=T^{-d / 2} \mathcal{P}(w)$ with $w=v T^{-1 / 2}$, or equivalently $F(y, \tau)=\Phi(x)$ with $x=y T$. The scaling function $\Phi(x)$ satisfies

$$
-\lambda x \Phi^{\prime}(x)+\Phi(x)=\langle\Phi(\xi x) \Phi(\eta x)\rangle
$$

and the boundary condition $\Phi(x)=1-\frac{1}{2} x+\cdots$ as $x \rightarrow 0$. In the elastic case, the velocity distribution is purely Maxwellian, $\Phi(x)=e^{-x / 2}$. Indeed, $\lambda=0$ and $\xi+\eta=1$ in this case. A stochastic process of elastic collisions effectively randomizes the velocities and leads to a thermal distribution [31]. In practice, this collision algorithm is used in Molecular dynamics simulations to thermalize velocities.

From the one-dimensional case, we anticipate that the velocity distribution has an algebraic large velocity tail. Generally, the large velocity behavior can be determined from the small wave number behavior of the Fourier transform. For example, the small- $x$ expansion of the one-dimensional solution (10)

\footnotetext{
${ }^{4}$ For elastic Maxwell molecules, the Fourier transform was first used in unpublished theses by Krupp [34], and then rediscovered and successfully utilized by Bobylev (see $[35,36]$ for a review).
} 


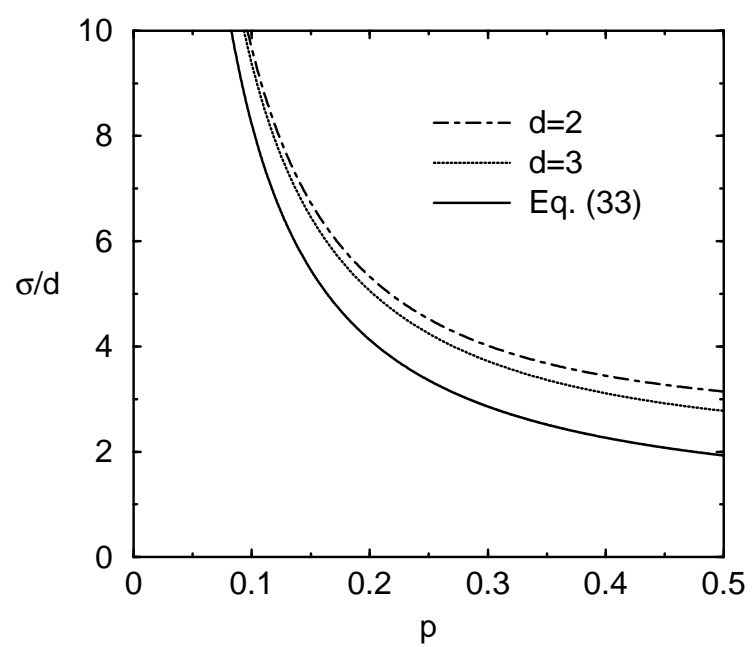

Fig. 1. The exponent $\sigma$ versus the dissipation parameter $p$ for $d=2$, and 3. For comparison with the leading large-dimension behavior (33), $\sigma$ is rescaled by $d$.

contains both regular and singular terms: $\Phi(x)=1-\frac{1}{2} x+\frac{1}{3} x^{3 / 2}+\cdots$, and the dominant singular $x^{3 / 2}$ term reflects the $w^{-4}$ tail of $\mathcal{P}(w)$. In general, if $\mathcal{P}(w)$ has an algebraic tail,

$$
\mathcal{P}(w) \sim w^{-\sigma} \quad \text { as } \quad w \rightarrow \infty,
$$

then $\Phi(x) \propto \int_{0}^{\infty} d w w^{d-1} \mathcal{P}(w) e^{i w \sqrt{x}}$ contains, apart from regular terms, the following dominant singular term: $\Phi_{\text {sing }}(x) \sim x^{(\sigma-d) / 2}$ as $x \rightarrow 0$. The exponent $\sigma$ can be now obtained by inserting $\Phi(x)=\Phi_{\text {reg }}(x)+\Phi_{\text {sing }}(x)$ into Eq. (30) and balancing the dominant singular terms. We find that $\sigma$ is a root of the integral equation ${ }^{5}$

$$
1-\lambda \frac{\sigma-d}{2}=\left\langle\xi^{(\sigma-d) / 2}+\eta^{(\sigma-d) / 2}\right\rangle .
$$

This equation can be recast as the eigenvalue problem $\lambda_{\nu}=\nu \lambda_{1}$ using $\lambda_{\nu}=\left\langle 1-\xi^{\nu}-\eta^{\nu}\right\rangle$ and $\nu=(\sigma-d) / 2$. It can also be expressed using the hypergeometric function ${ }_{2} F_{1}(a, b ; c ; z)[63]$ and the Euler's gamma function:

$1-p(1-p) \frac{\sigma-d}{d}={ }_{2} F_{1}\left[\frac{d-\sigma}{2}, \frac{1}{2} ; \frac{d}{2} ; 1-p^{2}\right]+(1-p)^{\sigma-d} \frac{\Gamma\left(\frac{\sigma-d+1}{2}\right) \Gamma\left(\frac{d}{2}\right)}{\Gamma\left(\frac{\sigma}{2}\right) \Gamma\left(\frac{1}{2}\right)}$.

Clearly, the exponent $\sigma \equiv \sigma(d, p)$ depends in a nontrivial fashion on the spatial dimension $d$ as well as the dissipation parameter $p$.

${ }^{5}$ This result was derived independently in $[44,47]$. 
There are two limiting cases where the velocity distribution approaches a Maxwellian, thereby implying a divergence of the exponent $\sigma$. In the quasielastic limit $(p \rightarrow 0)$ we have $\sigma \rightarrow d / p$. Even a minute degree of dissipation strongly changes the character of the system, and hence, energy dissipation is a singular perturbation $[24,29]$. As the dimension increases, the relative weight of the impact direction diminishes, and so does the role of dissipation. For large dimensions, the integral $\left\langle\eta^{(\sigma-d) / 2}\right\rangle$ in Eq. (32) vanishes exponentially and asymptotic analysis of the remaining integral shows that the exponent grows linearly with the dimension:

$$
\sigma \rightarrow d \frac{1+\frac{3}{2} p-p^{3}-p^{1 / 2}\left(1+\frac{5}{4} p\right)^{1 / 2}}{p\left(1-p^{2}\right)}
$$

when $d \rightarrow \infty$ [44]. Equation (33) provides a decent approximation even at moderate dimensions (Fig. 1). Overall, the exponent $\sigma(d, p)$ increases monotonically with increasing $d$, and additionally, it increases monotonically with decreasing $p$. Both features are intuitive as they mirror the monotonic dependence of the energy dissipation rate $\lambda=2 p(1-p) / d$ on $d$ and $p$. Remarkably, the exponent is very large. The completely inelastic case provides a lower bound for the exponent, $\sigma(d, p) \geq \sigma(d, 1 / 2)$ with $\sigma(d, 1 / 2)=6.28753$, 8.32937 , for $d=2,3$, respectively. For typical granular particles, $\sigma(d=3, p=$ $0.1) \cong 30$, and such algebraic decays are impossible to measure in practice.

The algebraic tail of the velocity distribution implies that moments of the scaling function $\Phi(x)$ with sufficiently large indices diverge. In the scaling regime, moments of the velocity distribution can be calculated by expanding the Fourier transform in powers of $x$,

$$
\Phi(x) \cong \sum_{n=0}^{[\nu]} \phi_{n}(-x)^{n} .
$$

The order of terms in this expansion must be smaller than the order of the singular term, $x^{\nu}$ [47]. The coefficients $\phi_{n}$, needed for calculating transport coefficients [54], yield the leading asymptotic behavior of the velocity moments, $M_{k}(t)=\int d \boldsymbol{v} v^{k} P(\boldsymbol{v}, t)$, via the relation $(2 n) ! T^{n} \phi_{n} \simeq\left\langle\mu^{n}\right\rangle M_{2 n}$. Inserting the moment expansion into (30) yields a closed hierarchy of equations

$$
\left(\lambda_{n}-n \lambda_{1}\right) \phi_{n}=\sum_{m=1}^{n-1} \lambda_{m, n-m} \phi_{m} \phi_{n-m},
$$

with $\lambda_{n}=\left\langle 1-\xi^{n}-\eta^{n}\right\rangle$ and $\lambda_{m, l}=\left\langle\xi^{m} \eta^{l}\right\rangle$. Calculation of these coefficients requires the following integrals [45]

$$
\left\langle\mu^{n}\right\rangle=\frac{\Gamma\left(\frac{d}{2}\right) \Gamma\left(n+\frac{1}{2}\right)}{\Gamma\left(\frac{1}{2}\right) \Gamma\left(n+\frac{d}{2}\right)}=\frac{1}{d} \frac{3}{d+2} \cdots \frac{2(n-1)+1}{2(n-1)+d} .
$$


Of course, $\phi_{0}=1$ and $\phi_{1}=1 / 2$; further coefficients can be determined recursively from (35), e.g.,

$$
\phi_{2}=\frac{1}{8} \frac{1-3 \frac{1-p^{2}}{d+2}}{1-3 \frac{1+p^{2}}{d+2}} .
$$

This coefficient is finite only when $\lambda_{2}>2 \lambda_{1}$ or $d<d_{2}=1+3 p^{2}$. Generally $\phi_{n}$ is finite only when the left hand side of Eq. (35) is positive, $\lambda_{n}>n \lambda_{1}$, or equivalently, when the dimension is sufficiently small, $d<d_{n}$. The crossover dimensions $d_{n}$ are determined from $\lambda_{n}=n \lambda_{1}$.

For $d>d_{n}$, moments with index smaller than $2 n$ are characterized by the temperature, while higher moments exhibit multiscaling asymptotic behavior. The time evolution of the moments can be studied using the expansion

$$
F(y, \tau)=\sum_{n=0}^{\infty} f_{n}(\tau)(-y)^{n} .
$$

The actual moments are related to the $f_{n}$ coefficients via $(2 n) ! f_{n}=\left\langle\mu^{n}\right\rangle M_{2 n}$. Substituting the expansion (37) into (29) yields the evolution equations

$$
\frac{d}{d \tau} f_{n}+\lambda_{n} f_{n}=\sum_{m=1}^{n-1} \lambda_{m, n-m} f_{m} f_{n-m} .
$$

We have $f_{1} \propto e^{-\lambda_{1} \tau}$, this is just the Haff's law. From $d f_{2} / d \tau+\lambda_{2} f_{2}=\lambda_{1,1} f_{1}^{2}$ we see that $f_{2}$ is a linear combination of two exponentials, $e^{-\lambda_{2} \tau}$ and $e^{-2 \lambda_{1} \tau}$. The two decay coefficients are equal $\lambda_{2}=2 \lambda_{1}$ at the crossover dimension $d=d_{2}$. As expected, when $d<d_{2}$ the fourth moment is dictated by the second moment, $f_{2} \propto f_{1}^{2}$. Otherwise, $f_{2} \propto \exp \left(-\lambda_{2} \tau\right)$. In general,

$$
M_{2 n} \propto \begin{cases}\exp \left(-n \lambda_{1} \tau\right) & d<d_{n} \\ \exp \left(-\lambda_{n} \tau\right) & d>d_{n}\end{cases}
$$

Fixing the dimension and the dissipation parameter, moments of sufficiently high order exhibit multiscaling asymptotic behavior. In practice, the exponent $\sigma$ is large, and multiscaling occurs only for very high order moments.

\subsection{The forced case}

We consider white noise forcing as in the one-dimensional case. The steady state Fourier transform, $F_{\infty}(k) \equiv F_{\infty}\left(k^{2}\right)$, satisfies

$$
\left(1+D k^{2}\right) F_{\infty}\left(k^{2}\right)=\left\langle F_{\infty}\left(\xi k^{2}\right) F_{\infty}\left(\eta k^{2}\right)\right\rangle .
$$

This equation is solved recursively by employing the cumulant expansion (24). Writing $1+D k^{2}=\exp \left[-\sum_{n \geq 1}\left(-D k^{2}\right)^{n} / n\right]$, we recast Eq. (40) into

$$
1=\left\langle\exp \left[-\sum_{n=1}^{\infty}\left(\widetilde{\psi}_{n}-n^{-1}\right)\left(-D k^{2}\right)^{n}\right]\right\rangle,
$$


with the auxiliary variables $\widetilde{\psi}_{n}=\psi_{n}\left(1-\xi^{n}-\eta^{n}\right)$. The coefficients $\psi_{n}$ are obtained by evaluating recursively the angular integrals of the auxiliary variables, $\left\langle\widetilde{\psi}_{n}\right\rangle$, and then using the identities $\psi_{n}=\left\langle\widetilde{\psi}_{n}\right\rangle / \lambda_{n}$. The few first coefficients can be determined explicitly, e.g., $\psi_{1}=1 / \lambda$ and

$$
\psi_{2}=\frac{3 d^{2}}{4(d+2)\left(1-p^{2}\right)-12(1-p)^{2}\left(1+p^{2}\right)} .
$$

The nonvanishing second cumulant shows the steady state distribution is not Maxwellian. Moreover, the poles of the Fourier transform located at $k=$ $\pm i / \sqrt{D}$ indicate that the large velocity tail of the distribution is exponential as in the one-dimensional case (21). Exponential decay is also suggested by the heuristic argument detailed above.

\subsection{Velocity correlations}

Maxwellian velocity distributions were originally obtained for random elastic collision processes (see Ref. [32], p. 36). Maxwell's seminal derivation involves two basic assumptions: (1) The velocity distribution is isotropic, and (2) Correlations between the velocity components are absent. In inelastic gases, the velocity distributions are non-Maxwellian - therefore, there must be correlations between the velocity components ${ }^{6}$. The quantity

$$
U=\frac{\left\langle v_{x}^{2} v_{y}^{2}\right\rangle-\left\langle v_{x}^{2}\right\rangle\left\langle v_{y}^{2}\right\rangle}{\left\langle v_{x}^{2}\right\rangle\left\langle v_{y}^{2}\right\rangle}
$$

provides a natural correlation measure. A non-vanishing $U$ indicates that velocity correlations do exist, and the larger $U$ the larger the correlation.

To compute $U$, we apply the identities:

$$
\left\langle v_{x}^{2}\right\rangle=\left.\frac{\partial^{2}}{\partial k_{x}^{2}} F\right|_{\boldsymbol{k}=\mathbf{0}} \quad \text { and } \quad\left\langle v_{x}^{2} v_{y}^{2}\right\rangle=\left.\frac{\partial^{2}}{\partial k_{x}^{2}} \frac{\partial^{2}}{\partial k_{y}^{2}} F\right|_{\boldsymbol{k}=\mathbf{0}} .
$$

The correlation measure is directly related to the fourth cumulant of the isotropic velocity distribution. In the freely cooling case, $U=8 \phi_{2}-1$; in the forced case, $U=\psi_{2} / \psi_{1}^{2}$. Substituting the corresponding coefficients yields

$$
\begin{aligned}
U_{\text {cooling }} & =\frac{6 p^{2}}{d-\left(1+3 p^{2}\right)}, \\
U_{\text {forced }} & =\frac{6 p^{2}(1-p)}{(d+2)(1+p)-3(1-p)\left(1+p^{2}\right)} .
\end{aligned}
$$

Generally, correlations increase monotonically with $p$. Correlations are much weaker in the presence of an energy source because the nature of the driving

\footnotetext{
${ }^{6}$ Inelastic collisions discriminate the impact direction, thereby generating correlations among the velocity components.
} 


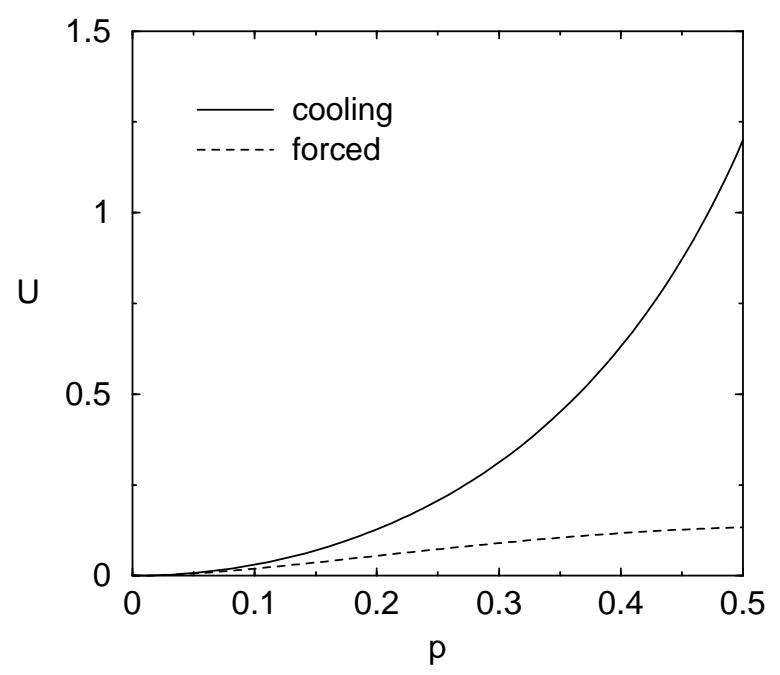

Fig. 2. The correlation measure $U$ for spatial dimension $d=3$. Shown are the freely cooling case and the forced case.

is random (Fig. 2). The perfectly inelastic case provides an upper bound. For example, when $d=3$ and $p=1 / 2$ we have $U_{\text {cooling }}=6 / 5$ and $U_{\text {forced }}=2 / 15$. Correlations decay as $U \propto p^{2}$ and $U \propto d^{-1}$ in the respective limiting cases of $p \rightarrow 0$ and $d \rightarrow \infty$, where Maxwellian distributions are recovered.

\section{Impurities}

The dynamics of an impurity immersed in a uniform granular fluid often defies intuition. The impurity does not generally behave as a tracer particle, and instead it may move either faster or slower than the fluid. Despite extensive studies, many theoretical and experimental questions regarding the dynamics of impurities remain open [64-72].

Impurities represent the simplest form of polydispersity, an important characteristic of granular media [73, 74]. Theoretically, the impurity problem is a natural first step in the study of mixtures as it involves fewer dissipation parameters. The fluid background is not affected by the presence of the impurity, and the impurity can be seen as "enslaved" to the fluid background.

We study dynamics of a single impurity particle in a uniform background of identical inelastic particles. We set the fluid particle mass to unity and the impurity mass to $m$. Collisions between two fluid particles are characterized by the dissipation parameter $p$ as in Eq. (25), while collisions between the impurity and any fluid particle are characterized by the dissipation parameter $q$. When an impurity particle of velocity $\boldsymbol{u}_{1}$ collides with a fluid particle of 
velocity $\boldsymbol{u}_{2}$ its post-collision velocity $\boldsymbol{v}_{1}$ is given by

$$
\boldsymbol{v}_{1}=\boldsymbol{u}_{1}-(1-q)(\boldsymbol{g} \cdot \boldsymbol{n}) \boldsymbol{n} .
$$

Two restitution coefficients, $r_{p} \equiv r=1-2 p$ and $r_{q}=m-(m+1) q$, characterize fluid-fluid and impurity-fluid collisions, respectively. The restitution coefficients obey $0 \leq r \leq 1$, and the dissipation parameters accordingly satisfy $0 \leq p \leq 1 / 2$ and $\frac{m-1}{m+1} \leq q \leq \frac{m}{m+1}$. The energy dissipated in an impurity-fluid collision is $\Delta E=-m(1-q)[2-(m+1)(1-q)](\boldsymbol{g} \cdot \boldsymbol{n})^{2}$.

Let $Q(\boldsymbol{v}, t)$ be the normalized velocity distribution of the impurity. In a random collision process, the impurity velocity distribution evolves according to the linear Lorentz-Boltzmann equation

$$
\begin{aligned}
\frac{\partial Q(\boldsymbol{v}, t)}{\partial t}= & K_{q} \int d \boldsymbol{n} \int d \boldsymbol{u}_{1} Q\left(\boldsymbol{u}_{1}, t\right) \int d \boldsymbol{u}_{2} P\left(\boldsymbol{u}_{2}, t\right) \\
& \times\left\{\delta\left[\boldsymbol{v}-\boldsymbol{u}_{1}+(1-q)(\boldsymbol{g} \cdot \boldsymbol{n}) \boldsymbol{n}\right]-\delta\left(\boldsymbol{v}-\boldsymbol{u}_{1}\right)\right\},
\end{aligned}
$$

while the fluid distribution obeys (26). Two rates, $K_{p} \equiv K$ and $K_{q}$, characterize fluid-fluid and fluid-impurity collisions, respectively. These rates can be eliminated from the respective equations (26) and (47) by introducing the collision counters, $\tau_{p} \equiv \tau=\int_{0}^{t} d t^{\prime} K_{p}\left(t^{\prime}\right)$, and $\tau_{q}=\int_{0}^{t} d t^{\prime} K_{q}\left(t^{\prime}\right)$. The Lorentz-Boltzmann equation is further simplified by using the Fourier transform, $G(\boldsymbol{k}, t)=\int d \boldsymbol{v} e^{i \boldsymbol{k} \cdot \boldsymbol{v}} Q(\boldsymbol{v}, t)$

$$
\frac{\partial}{\partial \tau_{q}} G(\boldsymbol{k})+G(\boldsymbol{k})=\int d \boldsymbol{n} G(\boldsymbol{k}-\boldsymbol{q}) F(\boldsymbol{q}),
$$

with $\boldsymbol{q}=(1-q)(\boldsymbol{k} \cdot \boldsymbol{n}) \boldsymbol{n}$. This equation supplements the fluid equation (27).

We consider two versions of the Maxwell model: An idealized case with equal collision rates, $K_{p}=K_{q}$ (model A); and a more physical case with collision rates proportional to appropriate average relative velocities (model B). In the forced case, one can obtain the impurity velocity distribution using the cumulant expansion, and generally, velocity statistics of the impurity are similar to the background. Below, we discuss the more interesting freely cooling case.

\subsection{Model A}

When the two collision rates are equal, all pairs of particles are equally likely to collide with each other. For simplicity we set the overall rate to unity, $K_{p}=K_{q}=1$; then both collision counters equal time, $\tau_{p}=\tau_{q}=t$.

In the freely cooling case, the fluid temperature decays exponentially, $T(t)=T_{0} \exp [-2 p(1-p) t / d]$. The impurity temperature, $\Theta(t)$, defined by $\Theta(t)=\frac{1}{d} \int d \boldsymbol{v} v^{2} Q(\boldsymbol{v}, t)$, is coupled to the fluid temperature via a linear rate equation

$$
\frac{d}{d t} \Theta=-\frac{1-q^{2}}{d} \Theta+\frac{(1-q)^{2}}{d} T .
$$


The solution to this equation is a linear combination of two exponentials

$$
\Theta(t)=\left(\Theta_{0}-c T_{0}\right) e^{-\left(1-q^{2}\right) t / d}+c T_{0} e^{-2 p(1-p) t / d}
$$

The constant $c=(1-q)^{2} /\left[1-q^{2}-2 p(1-p)\right]$ is simply the ratio between the impurity temperature and the fluid temperature in the long time limit. This generalizes the elastic fluid result $c=(1-q) /(1+q)[75,76]$. Generally, the impurity and the fluid have different energies, and this lack of equipartition is typical to granular particles $[6,77]$

There are two different regimes of behavior. When $1-q^{2}>2 p(1-p)$, the impurity temperature is proportional to the fluid temperature asymptotically, $\frac{\Theta(t)}{T(t)} \rightarrow c$ as $t \rightarrow \infty$. In the complementary region $2 p(1-p)>1-q^{2}$ the ratio of the fluid temperature to the impurity temperature vanishes. Since the system is governed by three parameters $\left(m, r_{p}, r_{q}\right)$, it is convenient to consider the restitution coefficients as fixed and to vary the impurity mass. From the definition of the restitution coefficients the borderline case, $q^{2}=1-2 p(1-p)$, defines a critical impurity mass

$$
m_{*}=\frac{r_{q}+\sqrt{\left(1+r_{p}^{2}\right) / 2}}{1-\sqrt{\left(1+r_{p}^{2}\right) / 2}} .
$$

This critical mass is always larger than unity. Asymptotically, the impurity to fluid temperature ratio exhibits two different behaviors

$$
\frac{\Theta(t)}{T(t)} \rightarrow \begin{cases}c & m<m_{*} \\ \infty & m \geq m_{*}\end{cases}
$$

We term these two regimes, the light impurity phase and the heavy impurity phase, respectively. In the light impurity phase, the initial impurity temperature becomes irrelevant asymptotically, and the impurity is governed by the fluid background. In the heavy impurity phase, the impurity is infinitely more energetic compared with the fluid and practically, it sees a static fluid. A qualitatively similar phase transition is observed for impurities in inelastic hard-spheres [71,72]. Interestingly, the dependence on the dimension is secondary as both the critical mass, $m_{*}$, and the temperature ratio $c$ are independent of $d$. Below we study velocity statistics including the velocity distribution and its moments primarily in one dimension, where explicit solutions are possible.

The light impurity phase In this phase, $m<m_{*}$, the impurity is governed by the fluid background. We therefore seek scaling solutions of the same form as the fluid's: $Q(v, t)=T^{-1 / 2} \mathcal{Q}\left(v T^{-1 / 2}\right)$ and $G(k, t)=g\left(|k| T^{1 / 2}\right)$. From the Lorentz-Boltzmann equation (48), the latter scaling function satisfies the linear equation

$$
-p(1-p) z g^{\prime}(z)+g(z)=g(q z)[1+(1-q) z] e^{-(1-q) z} .
$$


Since the fluid scaling function is a combination of $z^{n} e^{-z}$ with $n=0$ and $n=1$, we try the series ansatz

$$
g(z)=\sum_{n=0}^{\infty} g_{n} z^{n} e^{-z}
$$

for the impurity. The first few coefficients, $g_{0}=g_{1}=1$ and $g_{2}=(1-c) / 2$, follow from the small- $z$ behavior $g(z) \cong 1-\frac{1}{2} c z^{2}$. The rest are obtained recursively

$$
g_{n}=\frac{q^{n-1}(1-q)-p(1-p)}{1-q^{n}-n p(1-p)} g_{n-1} .
$$

The Fourier transform can be inverted to obtain the impurity velocity distribution function explicitly. The inverse Fourier transform of $e^{-\kappa z}$ is $\frac{1}{\pi} \frac{\kappa}{\kappa^{2}+w^{2}}$; the inverse transforms of $z^{n} e^{-z}$ can be obtained using successive differentiation with respect to $\kappa$. Therefore, the solution is a series of powers of Lorentzians

$$
\mathcal{Q}(w)=\frac{2}{\pi} \sum_{n=2}^{\infty} Q_{n} \frac{1}{\left(1+w^{2}\right)^{n}}
$$

The coefficients $Q_{n}$ are linear combinations of the coefficients $g_{k}$ 's with $k \leq$ $n+1$, e.g., $Q_{2}=1-3 g_{2}+3 g_{3}$ and $Q_{3}=4 g_{2}-24 g_{3}+60 g_{4}$. There are special values of $q$ for which the infinite sum terminates at a finite order. Of course, when $q=p$, the impurity is identical to the fluid and $Q_{n}=0$ for all $n>2$. When $q^{2}(1-q)=p(1-p)$, one has $Q_{n}=0$ for all $n>3$, so the velocity distribution includes only the first two terms. Regardless of $q$, the first squared Lorentzian term dominates the tail of the velocity distribution $\mathcal{Q}(w) \sim \mathcal{P}(w) \sim w^{-4}$, as $w \rightarrow \infty$. One can show that this behavior extends to higher dimensions, $\mathcal{Q}(w) \sim \mathcal{P}(w) \sim w^{-\sigma}$. Therefore, the impurity has the same algebraic extremal velocity statistics as the fluid.

While the scaling functions underlying the impurity and the fluid are similar, more subtle features may differ. Moments of the impurity velocity distribution, $L_{n}(t)=\int d v v^{n} Q(v, t)$, obey the recursive equations

$$
\frac{d}{d t} L_{n}+b_{n} L_{n}=\sum_{m=2}^{n-2}\left(\begin{array}{l}
n \\
m
\end{array}\right) q^{m}(1-q)^{n-m} L_{m} M_{n-m}
$$

with $b_{n}(q)=1-q^{n}$. Asymptotically, the fluid moments decay exponentially according to $M_{n}(t) \propto e^{-a_{n}(p) t}$. Using this asymptotic behavior, we analyze the (even) impurity moments. The second moment, i.e. the impurity temperature, was shown to behave similar to the fluid temperature when $a_{2}(p)<b_{2}(q)$. The fourth moment behaves similarly to the fourth moment of the fluid, $L_{4} \propto M_{4}$, when $a_{4}(p)<b_{4}(q)$. However, in the complementary case, the fourth moment behaves differently, $L_{n}(t) \propto e^{-b_{4}(q) t}$. In general, when $a_{n}(p)<b_{n}(q)$, the $n$th impurity moment is proportional to the $n$th 
fluid moment, $L_{n} \propto M_{n}$. Otherwise, when $a_{n}(p)>b_{n}(q)$, the $n$th impurity moment is no longer governed by the fluid and

$$
L_{n}(t) \propto e^{-\left(1-q^{n}\right) t} .
$$

This series of transition affecting moments of decreasing order occurs at increasing impurity masses,

$$
m_{1}>m_{2}>\cdots>m_{\infty} .
$$

When $m \geq m_{n}$, the ratio $M_{2 k} / L_{2 k}$ diverges asymptotically for all $k \geq n$. The transition masses

$$
m_{n}=\frac{r_{q}+\frac{1}{2}\left[\left(1-r_{p}\right)^{2 n}+\left(1+r_{p}\right)^{2 n}\right]^{\frac{1}{2 n}}}{1-\frac{1}{2}\left[\left(1-r_{p}\right)^{2 n}+\left(1+r_{p}\right)^{2 n}\right]^{\frac{1}{2 n}}}
$$

are found from $q^{2 n}=p^{2 n}+(1-p)^{2 n}$ and the definitions of the restitution coefficients. All of the transition masses are larger than unity, so the impurity must be heavier than the fluid for any transition to occur. The largest transition mass is $m_{1} \equiv m_{*}$, and the smallest transition mass is $m_{\infty}=\lim _{n \rightarrow \infty} m_{n}=\left(1+r_{p}+2 r_{q}\right) /\left(1-r_{p}\right)$. Impurities lighter than the latter mass, $m<m_{\infty}$, mimic the fluid completely.

The heavy impurity phase In this phase, $m>m_{*}$, the velocities of the fluid particles are asymptotically negligible compared with the velocity of the impurity. Fluid particles become stationary as viewed by the impurity, and effectively, $\boldsymbol{u}_{2} \equiv 0$ in the collision rule (46):

$$
\boldsymbol{v}=\boldsymbol{u}-(1-q)(\boldsymbol{u} \cdot \boldsymbol{n}) \boldsymbol{n} .
$$

Mathematically, this process is reminiscent of a Lorentz gas [78]. Physically, the two processes are different. In the granular impurity system, a heavy particle scatters of a static background of lighter particles, while in the Lorentz gas the scatterers are infinitely massive.

We first consider the one-dimensional case. Setting $u_{2} \equiv 0$ in the LorentzBoltzmann equation (47), integration over the fluid velocity $u_{2}$ is trivial, $\int d u_{2} P\left(u_{2}, t\right)=1$, and integration over the impurity velocity $u_{1}$ gives

$$
\frac{\partial}{\partial t} Q(v, t)+Q(v, t)=\frac{1}{q} Q\left(\frac{v}{q}, t\right) .
$$

This equation can be solved directly by considering the stochastic process the impurity particle experiences. In a sequence of collisions, the impurity velocity changes according to $v_{0} \rightarrow q v_{0} \rightarrow q^{2} v_{0} \rightarrow \cdots$ with $v_{0}$ the initial velocity. After $n$ collisions the impurity velocity decreases exponentially, $v_{n}=q^{n} v_{0}$. Furthermore, the collision process is random, and therefore, the probability 
that the impurity undergoes exactly $n$ collisions up to time $t$ is Poissonian $t^{n} e^{-t} / n$ !. Thus, the velocity distribution function reads

$$
Q(v, t)=e^{-t} \sum_{n=0}^{\infty} \frac{t^{n}}{n !} \frac{1}{q^{n}} Q_{0}\left(\frac{v}{q^{n}}\right),
$$

where $Q_{0}(v)$ is the initial velocity distribution of the impurity. The impurity velocity distribution function is a time-dependent combination of "replicas" of the initial velocity distribution. Since the arguments are stretched, compact velocity distributions display an infinite set of singularities, a generic feature of the Maxwell model.

In contrast to the velocity distribution, the moments $L_{n}(t)$ exhibit a much simpler behavior. Indeed, from Eq. (62) one finds that every moment is coupled only to itself, $\frac{d}{d t} L_{n}=-\left(1-q^{n}\right) L_{n}$. Solving this equation we recover Eq. (58); in the heavy impurity phase, however, it holds for all $n$. Therefore the moments exhibit multiscaling asymptotic behavior. The decay coefficients, characterizing the $n$-th moment, depend on $n$ in a nonlinear fashion.

\subsection{Model B}

For hard sphere particles, the collision rates are proportional to the relative velocity. The overall collision rates in the Maxwell model represent the aver-

age relative velocity, and a natural choice is $\sqrt{\left\langle\left(\boldsymbol{v}_{1}-\boldsymbol{v}_{2}\right)^{2}\right\rangle} \propto \sqrt{\left(T_{1}+T_{2}\right) / 2}$. Therefore, the rates $K_{p}=\sqrt{T}$ and $K_{q}=\sqrt{(T+\Theta) / 2}$, should be used in the Boltzmann equation (26) and the Lorentz-Boltzmann equation (47), respectively. This modification suppresses the heavy impurity phase, although the secondary transitions corresponding to higher order moments remain.

The fluid and the impurity temperatures obey

$$
\begin{aligned}
\frac{d}{d t} T & =-\sqrt{T}\left[\frac{2 p(1-p)}{d} T\right], \\
\frac{d}{d t} \Theta & =\sqrt{\frac{T+\Theta}{2}}\left[-\frac{1-q^{2}}{d} \Theta+\frac{(1-q)^{2}}{d} T\right] .
\end{aligned}
$$

Consequently, the temperature ratio, $S=\Theta / T$, evolves according to

$$
\frac{1}{\sqrt{T}} \frac{d}{d t} S=\sqrt{\frac{1+S}{2}}\left[-\frac{1-q^{2}}{d} S+\frac{(1-q)^{2}}{d}\right]+\frac{2 p(1-p)}{d} S .
$$

The loss term, which grows as $S^{3 / 2}$, eventually overtakes the gain term that grows only linearly with $S$. Therefore, the asymptotic temperature ratio remains finite, $S \rightarrow c$, where $c$ is the root of the cubic equation

$$
\sqrt{\frac{1+c}{2}}\left(c-\frac{1-q}{1+q}\right)=\frac{2 p(1-p)}{1-q^{2}} c .
$$


Consequently, there is only one phase, the light impurity phase. Intuitively, as the impurity becomes more energetic, it collides more often with fluid particles. This mechanism limits the growth rate of the temperature ratio. As in model A, energy equipartition does not generally occur, and the behavior is largely independent of the spatial dimension.

Qualitatively, results obtained for the light impurity phase in model A extend to model B. The impurity velocity distribution follows a scaling asymptotic behavior. The only difference is that the collision terms are proportional to $\beta^{-1}=\sqrt{(1+c) / 2}$, and Eq. (53) generalizes as follows

$$
-\beta p(1-p) z g^{\prime}(z)+g(z)=(1+\alpha z) e^{-\alpha z} g(q z) .
$$

Seeking a series solution of the form (54) leads to the following recursion relations for the coefficients

$$
g_{n}=\frac{(1-q) q^{n-1}-\beta p(1-p)}{1-q^{n}-n \beta p(1-p)} g_{n-1} .
$$

Again, the velocity distribution is a combination of powers of Lorentzians as in Eq. (56). Moreover, the coefficients $Q_{n}$ are linear combinations of the coefficients $g_{n}$ 's as in model A. Most importantly, the large-velocity tail is generic $\mathcal{Q}(w) \sim w^{-4}$.

The fluid moments evolve according to (11) with $\tau \equiv \tau_{p}$, while the impurity moments evolve according to

$$
\frac{d}{d \tau_{q}} L_{n}+b_{n} L_{n}=\sum_{m=2}^{n-2}\left(\begin{array}{c}
n \\
m
\end{array}\right) q^{m}(1-q)^{n-m} M_{m} L_{m-j},
$$

Note that asymptotically $\tau_{q} \rightarrow \tau_{p} / \beta$, so the fluid moments decay according to $M_{n} \propto e^{-\beta a_{n}(p) \tau_{q}}$. Hence, when $\beta a_{n}(p)<b_{n}(q)$, the impurity moments are enslaved to the fluid moments, i.e., $L_{n} \propto M_{n}$ asymptotically. Otherwise, sufficiently large impurity moments behave differently than the fluid moments, viz. $M_{n} \propto e^{-b_{n} \tau_{q}}$. Although the primary transition affecting the second moment does not occur $\left(m_{1} \equiv m_{*}=\infty\right)$, secondary transitions affecting larger moments do occur at a series of masses, as in Eq. (59). The transition masses $m_{n}$ are found by solving $\beta a_{n}(p)=b_{n}(q)$ simultaneously with Eq. (65). For example, for completely inelastic collisions $\left(r_{p}=r_{q}=0\right)$ one finds $m_{2}=1.65$. These transitions imply that some velocity statistics of the impurity, specifically large moments, are no longer governed by the fluid.

\subsection{Velocity autocorrelations}

The impurity autocorrelation function satisfies $d A / d \tau_{q}=-(1-q) A$. Therefore, its decay is similar to (15)

$$
A\left(\tau_{q}^{\prime}, \tau_{q}\right) \sim A_{0} \exp \left[-(1-q)\left(\tau_{q}-\tau_{q}^{\prime}\right)\right]
$$


with $q$ replacing $p$ and $\tau_{q}$ replacing $\tau \equiv \tau_{p}$. For model $A$ where the collision counters equal time, the decay remains exponential. However, the impurity autocorrelation decays with a different rate than the fluid. For model B, one can show that the algebraic decay (16) holds asymptotically with $p$ replaced by $q$. We conclude that while one-point velocity statistics of the impurity are governed by the fluid, two-time statistics are different.

\section{$5 \quad$ Mixtures}

Granular media typically consists of mixtures of granular particles of several types. In contrast with the impurity problem, the different components of a mixture are coupled to each other. Moreover, there are numerous parameters, making mixtures much harder to treat analytically [51]. Remarkably, the impurity solution can be generalized to arbitrary mixtures ${ }^{7}$. We detail the freely cooling case in one dimension.

Consider a binary mixture where particles of type 1 have mass $m_{1}$ and concentration $c_{1}$, and similarly for particles of type 2 . We set $c_{1}+c_{2}=1$. Also, unit collision rates are considered for simplicity. Collisions between a particle of type $i=1,2$ and a particle of type $j=1,2$ are characterized by the dissipation parameter $p_{i j}=\left(m_{i}-m_{j} r_{i j}\right) /\left(m_{i}+m_{j}\right)$, with $r_{i j}$ the restitution coefficient. We denote the normalized velocity distribution of component $i$ by $P_{i}(v, t)$ and its Fourier transform by $F_{i}(k, t)$. The governing equations now couple the two distributions

$$
\frac{\partial}{\partial t} F_{i}(k)+F_{i}(k)=\sum_{j=1}^{2} c_{j} F_{j}\left(k-p_{i j} k\right) F_{i}\left(p_{i j} k\right) .
$$

Let $T_{i}=\int d v v^{2} P_{i}(v, t)$ be the temperature of the $i$ th component. Writing $F_{i}(k, t) \cong 1-\frac{1}{2} k^{2} T_{i}$, the temperatures evolve according

$$
\frac{d}{d t}\left(\begin{array}{l}
T_{1} \\
T_{2}
\end{array}\right)=-\left(\begin{array}{ll}
\lambda_{11} & \lambda_{12} \\
\lambda_{21} & \lambda_{22}
\end{array}\right)\left(\begin{array}{l}
T_{1} \\
T_{2}
\end{array}\right)
$$

The diagonal matrix elements are $\lambda_{i i}=2 c_{i} p_{i i}\left(1-p_{i i}\right)+c_{j}\left(1-p_{i j}^{2}\right)$ with $j \neq i$, and the off-diagonal matrix elements are $\lambda_{i j}=-c_{j}\left(1-p_{i j}\right)^{2}$. Therefore, the temperatures are sums of two exponential terms, $\exp \left(-\lambda_{ \pm} t\right)$. The decay coefficients are the two (positive) eigenvalues

$$
\lambda_{ \pm}=\frac{1}{2}\left[\lambda_{11}+\lambda_{22} \pm \sqrt{\left(\lambda_{11}-\lambda_{22}\right)^{2}+4 \lambda_{12} \lambda_{21}}\right] .
$$

Asymptotically, the term with the smaller decay rate $\lambda \equiv \lambda_{-}$dominates

$$
T_{i}(t) \simeq C_{i} e^{-\lambda t}, \quad \text { with } \quad C_{i}=\frac{\left(\lambda_{+}-\lambda_{i i}\right) T_{i}(0)-\lambda_{i j} T_{j}(0)}{\lambda_{+}-\lambda_{-}} .
$$

\footnotetext{
${ }^{7}$ Mixtures were treated analytically in the elastic Maxwell model $[79,80]$
} 
The ratio between the two temperatures approaches $C_{2} / C_{1}=\left(\lambda-\lambda_{11}\right) / \lambda_{12}$, and the two components have different temperatures. Furthermore, as long as the components are coupled, both temperatures are finite. For example, a vanishing $C_{2}$ implies that one of the concentrations vanishes as $\lambda=\lambda_{1,1}$.

As in the cases of homogeneous gases and impurities, we seek a scaling solution of the form $P_{i}(v, t)=e^{\lambda t / 2} \mathcal{P}_{i}(w)$ with $w=v e^{\lambda t / 2}$, or equivalently, $F_{i}(k, t)=f_{i}(z)$ with $z=|k| e^{-\lambda t / 2}$. The scaling functions $f_{i}(z)$ are coupled via the non-local differential equations

$$
-\frac{1}{2} \lambda z f_{i}(z)+f_{i}(z)=\sum_{j=1}^{2} c_{j} f_{j}\left(z-p_{i j} z\right) f_{i}\left(p_{i j} z\right) .
$$

Substituting the series solution (54), $f_{i}(z)=\sum_{n=0}^{\infty} A_{i, n} z^{n} e^{-z}$, yields recursion relations for the coefficients $A_{i, n}$

$$
\left(1-\frac{n \lambda}{2}\right) A_{i, n}+\frac{\lambda}{2} A_{i, n-1}=\sum_{j=1}^{2} \sum_{m=0}^{n} c_{j} A_{j, m} A_{i, n-m}\left(1-p_{i j}\right)^{m} p_{i j}^{n-m}
$$

The small $z$ behavior $f_{i}(z)=1-\frac{1}{2} C_{i} z^{2}$ implies the first three coefficients $A_{i, 0}=A_{i, 1}=1$, and $A_{i, 2}=\left(1-C_{i}\right) / 2$. For $n \geq 3$, the coefficients $\left(A_{1, n}, A_{2, n}\right)$ are solved recursively in pairs. Each such pair satisfies two inhomogeneous linear equations. Therefore, as in the impurity case, the velocity distribution is an infinite series of powers of Lorentzians (56). Although the two velocity distributions are different, they have the same extremal behavior, $\mathcal{P}_{i}(w) \sim w^{-4}$. It is straightforward to generalize the above to mixtures with arbitrary number of components, and to incorporate different collision rates, in particular, $K_{i j}=\sqrt{\left(T_{i}+T_{j}\right) / 2}$.

\section{Lattice Gases}

Inelastic collisions generate spatial correlations and consequently, inelastic gases exhibit spatial structures such as shocks. Thus far, we considered only mean-field collision processes where there is no underlying spatial structure. Random collision processes can be naturally generalized by placing particles on lattice sites and allowing only nearest neighbors to collide.

Consider a one-dimensional lattice where each site is occupied by a single particle. Let $v_{j}$ be the velocity of the particle at site $j$. The velocity of such a particle changes according to Eq. (1) due to interactions with either of its two neighbors. Time is conveniently characterized by the collision counter $\tau$. In an infinitesimal time interval $d \tau$, the velocity of a particle changes as follows

$$
v_{j}(\tau+d \tau)= \begin{cases}v_{j}(\tau) & \text { prob. } 1-2 d \tau ; \\ v_{j}(\tau)-(1-p)\left[v_{j}(\tau)-v_{j-1}(\tau)\right] & \text { prob. } d \tau ; \\ v_{j}(\tau)-(1-p)\left[v_{j}(\tau)-v_{j+1}(\tau)\right] & \text { prob. } d \tau .\end{cases}
$$


This process is stochastic and we are interested in averages over all possible realizations of the process, denoted by an overline. We consider random initial conditions where the average velocity vanishes and no correlations are present: $\overline{v_{j}(0)}=0$ and $\overline{v_{i}(0) v_{j}(0)}=T_{0} \delta_{i, j}$.

Spatial velocity correlations satisfy closed equations as in the Ising-Glauber spin model [81]. For example, from the dynamical rules (76), the average velocity $V_{j}(\tau)=\overline{v_{j}(\tau)}$, obeys a discrete diffusion equation

$$
\frac{d V_{j}}{d \tau}=(1-p)\left(V_{j-1}-2 V_{j}+V_{j+1}\right) .
$$

From the initial conditions, $V_{j}(0)=0$, we obtain $V_{j}(t)=0$. Consider the spatial correlation function $\overline{v_{i} v_{j}}$. The initial state is translationally invariant, so this property persists. The correlation functions $R_{n}=\overline{v_{j} v_{j+n}}$ satisfy

$$
\begin{aligned}
\frac{d R_{n}}{d \tau} & =2(1-p)\left(R_{n-1}-2 R_{n}+R_{n+1}\right), \quad n \geq 2 ; \\
\frac{d R_{1}}{d \tau} & =2(1-p)\left[p R_{0}-(1+p) R_{1}+R_{2}\right] ; \\
\frac{d R_{0}}{d \tau} & =4 p(1-p)\left[R_{1}-R_{0}\right] .
\end{aligned}
$$

The initial conditions are $R_{n}(0)=T_{0} \delta_{n, 0}$. Since we are interested in the asymptotic behavior, we employ the continuum approximation. The correlation function satisfies the diffusion equation $\partial R / \partial \tau=2(1-p) \partial^{2} R / \partial^{2} n$, so the solution is the Gaussian

$$
R_{n}(\tau) \simeq \frac{T_{0}}{\sqrt{8(1-p) \pi \tau}} \exp \left[-\frac{n^{2}}{8(1-p) \tau}\right]
$$

The temperature, $T \equiv R_{0}$ decays as $T(\tau) \simeq T_{0}[8(1-p) \pi \tau]^{-1 / 2}$ in the long time limit ${ }^{8}$.

Although no correlations were present in the initial conditions, spatial correlations develop at later times. The corresponding correlation length $\xi$ grows diffusively with the collision counter, $\xi \sim \tau^{1 / 2}$. The system consists of a network of domains of typical size $\xi$. Inside a domain, velocities are strongly correlated, and momentum conservation yields a relation between the average velocity and the domain size, $v_{*} \sim \xi^{-1 / 2} \sim \tau^{-1 / 4}$. This scale is consistent with the temperature behavior above, $T \sim v_{*}^{2}$. In arbitrary dimension, this scaling argument yields $T \sim \tau^{-d / 2}[22,43]$. At least for scalar velocities, the correlation functions obey closed equations in arbitrary dimension [82], and this behavior can be also obtained analytically.

The actual time dependence is determined from the collision rate. We consider two choices. In model $\mathrm{A}$, the rate is proportional to the typical velocity

\footnotetext{
8 An exact solution of the discrete equations (78) is possible. It yields an identical asymptotic expression for the temperature.
} 
$K^{2}=T$. In the physically more realistic model $\mathrm{B}$, the rate is proportional to the average relative velocity $K^{2}=\overline{\left(v_{j+1}-v_{j}\right)^{2}}=2\left(R_{0}-R_{1}\right) \propto-d T / d \tau$. Hence $K \sim \tau^{-\alpha}$ with $\alpha=d / 4$ (model A) and $\alpha=(d+2) / 4$ (model B). The time $t=\int_{0}^{\tau} d \tau^{\prime} / K\left(\tau^{\prime}\right)$ grows as $t \sim \tau^{1+\alpha}$, and therefore,

$$
T \sim t^{-\gamma} \quad \text { with } \quad \gamma= \begin{cases}\frac{2 d}{d+4} & \text { model A; } \\ \frac{2 d}{d+6} & \text { model B }\end{cases}
$$

In either case, Haff's cooling law is recovered only in the infinite dimension limit, $d \rightarrow \infty$. Otherwise, the appearance of spatial correlations slows down the temperature decay. As neighboring particles become correlated their relative velocity and consequently collision rate is reduced. Qualitatively similar behavior was shown for freely cooling inelastic gases, namely, breakdown of the mean-field cooling law due to the formation of strong spatial correlations among particles velocities $[24,25]$.

\section{Conclusions}

We presented analytic results for random inelastic collision processes. In general, when the collision rate is uniform, the convolution structure of the collision integrals translates to products in Fourier space. While the governing equations are both nonlinear and nonlocal, they are closed and amenable to analytical treatment. In the freely cooling case, a small wave number analysis of the Fourier transform displays both regular and singular terms. The regular terms yield the low order moments, while the leading singular term gives the high-energy tail. In the forced case, the Fourier transform is an analytic function and complex residue analysis yields the high-energy tail.

In the freely cooling case, the velocity distribution approaches a scaling form and displays an algebraic large velocity tail. In one dimension, the scaling function is a universal; otherwise, it depends on the degree of inelasticity. The exponent governing the high-energy tail is typically very large, and may be difficult to measure in practice. We have also shown that sufficiently large moments exhibit multiscaling behavior, and hence, are not characterized by the temperature. The autocorrelation function decays algebraically, with an exponent that depends on the dissipation parameters. The spread of a tagged particle exhibits a universal $\sqrt{\ln t}$ growth.

We demonstrated that an impurity immersed in a uniform fluid may or may not mimic the background. Fixing the dissipation parameters, for sufficiently low impurity mass, the impurity's velocity distribution, velocity moments and extremal velocity statistics are all governed by the fluid. However, there is a series of phase transitions occurring at a series of increasing masses, where impurity moments of decreasing orders decouple from the fluid. These transitions indicate that sufficiently heavy impurities are very energetic and effectively, they experience a static fluid background. 
For binary mixtures, we examined only primary velocity statistics and showed that qualitatively, all components have the same temperature decay and extremal velocity statistics. It remains to be seen whether the different components may exhibit different asymptotic behaviors of more subtle velocity statistics such as the high order moments. The impurity case demonstrates how in certain limiting cases, mixtures may display anomalous behavior.

In the forced case, injection of energy counters the energy dissipation and the system relaxes toward a steady state. We considered white noise forcing, and showed that the steady state distribution has an exponential high-energy tail. Steady state characteristics in the forced case are directly related to time dependent relaxation characteristics in the freely cooling case.

Results obtained in the framework of the inelastic Maxwell model are exact for random collision processes where spatial structure is absent, and particles collide irrespective of their relative velocities. Such results are an uncontrolled approximation of the inelastic hard sphere problem. Nevertheless, as a conceptual tool, the inelastic Maxwell model is powerful. For example, it demonstrates the development of correlations among the velocity components, as well as spatial correlations. It also raises doubts concerning the suitability of several widely used techniques such as perturbation expansions in the quasi-elastic limit, and expansions in terms of Sonine polynomials.

We demonstrated that a lattice gas generalization of the Maxwell model remains tractable when the kinematic constraint (particles should collide only if they are moving toward each other) is ignored. Taking this constraint into account leads to shock-like structures in one dimension and to vortices in two dimensions [43]. Lattice models can therefore be used to study development of spatial correlations and spatial structures in inelastic gases.

We presented in detail the most basic realization of the Maxwell model. Several other generalizations are feasible. Experiments can now measure the distributions of impact angles and of the effective restitution coefficients. Such phenomenological information can be incorporated into the rate equations. The integration measure can be redefined to give different weights to different angles. Moreover, a distribution of restitution coefficients can be introduced by integrating the collision integrals with respect to the dissipation parameters. For example, random collision processes were successfully used to model the role of the boundary in driven gases [83].

We restricted our attention to the case where all moments of the initial velocity distribution are finite. However, there are other infinite energy solutions of the Boltzmann equation. For example, any Lorentzian, $F(k)=\exp (-C k)$, is a steady state solution of Eq. (6). A remaining challenge is to classify the evolution of an arbitrary velocity distribution. Also, it will be interesting to characterize the full spectrum of extremal behaviors, characterizing velocities far larger than the typical velocity.

Finally, to model transport and other hydrodynamics problems one has to incorporate the spatial dependence explicitly. In the framework of the 
Maxwell model the simplest such problem - the shear flow - has been recently investigated by Cercignani [84]. For inelastic hard spheres, a number of unidirectional hydrodynamic flows were analyzed by Goldshtein and coworkers [85-87]. It would be interesting to investigate these problems in the framework of the Maxwell model.

Acknowledgments We have benefited from discussions and correspondence with A. Baldassarri and M. H. Ernst. We also thank N. Brilliantov, A. Goldshtein, A. Puglisi, S. Redner, and H. A. Rose for useful discussions. This research was supported by DOE (W-7405-ENG-36) and NSF(DMR9978902).

\section{References}

1. T. Pöschel and S. Luding (editors), Granular Gases (Springer, Berlin, 2000).

2. H. M. Jaeger, S. R. Nagel, and R. P. Behringer, Rev. Mod. Phys. 68, 1259 (1996).

3. W. Losert, D. G. W. Cooper, J. Delour, A. Kudrolli, and J. P. Gollub, Chaos 9, 682 (1999).

4. J.S. Olafsen and J.S. Urbach, Phys. Rev. E 60, R2468 (1999).

5. F. Rouyer and N. Menon, Phys. Rev. Lett. 85, 3676 (2000).

6. R. D. Wildman and D. J. Parker, Phys. Rev. Lett. 88, 064301 (2002).

7. A. Kudrolli, M. Wolpert and J. P. Gollub, Phys. Rev. Lett. 78, 1383 (1997).

8. A. Samadani, L. Mahadevan, and A. Kudrolli, J. Fluid Mech. 452, 293 (2002).

9. E. Rericha, C. Bizon, M. D. Shattuck, and H. L. Swinney, Phys. Rev. Lett. 88, $014302(2002)$.

10. K. M. Hill and J. Kakalios, Phys. Rev. E R3610 (1996).

11. F. Mello, P. B. Umbanhowar, and H. L. Swinney, Phys. Rev. Lett. 75, 3838 (1995).

12. J. S. Olafsen and J. S. Urbach, Phys. Rev. Lett. 81, 4369 (1998).

13. H. J. Herrmann, in: Disorder and Granular Media, edited by D. Bideau and A. Hansen (Elsevier Science Publishers, 1993).

14. D. R. M. Williams and F. C. MacKintosh, Phys. Rev. E 54, R9 (1996).

15. E. L. Grossman, T. Zhou, and E. Ben-Naim, Phys. Rev. E 55, 4200 (1997).

16. C. Bizon, M. D. Shattuck, J. B. Swift, W. D. McCormick, and H. L. Swinney, Phys. Rev. Lett. 80, 57 (1998).

17. B. Bernu and R. Mazighi, J. Phys. A 23, 5745 (1990).

18. S. McNamara and W. R. Young, Phys. Fluids A 4, 496 (1992); Phys. Rev. E 50, R28 (1994).

19. T. Zhou and L. P. Kadanoff, Phys. Rev. E 54, 623 (1996).

20. Y. Du, H. Li, and L. P. Kadanoff, Phys. Rev. Lett. 74, 1268 (1995).

21. I. Goldhirsch and G. Zanetti, Phys. Rev. Lett. 70, 1619 (1993).

22. R. Brito and M. H. Ernst, Europhys. Lett. 43, 497 (1998).

23. M. R. Swift, M. Boamfă, S. J. Cornell, and A. Maritan, Phys. Rev. Lett. 80, 4410 (1998).

24. E. Ben-Naim, S. Y. Chen, G. D. Doolen, and S. Redner, Phys. Rev. Lett. 83, 4069 (1999).

25. X. Nie, E. Ben-Naim, and S. Y. Chen, Phys. Rev. Lett. 89, 204301 (2002). 
26. S. E. Esipov and T. Pöschel, J. Stat. Phys. 86, 1385 (1997).

27. P. Deltour and A. Barrat, J. Phys. I France 7, 137 (1997).

28. T. P. C. van Noije and M. H. Ernst, Granular Matter 1, 57 (1998).

29. A. Puglisi, V. Loreto, U. M. B. Marconi, and A. Vulpiani, Phys. Rev. E 59, $5582(1999)$.

30. A. Barrat, T. Biben, Z. Rácz, E. Trizac, and F. van Wijland, J. Phys. A 35, 463 (2002).

31. J. C. Maxwell, Phil. Trans. R. Soc. 157, 49 (1867).

32. P. Résibois and M. de Leener, Classical Kinetic Theory of Fluids (John Wiley, New York, 1977).

33. C. Truesdell and R. G. Muncaster, Fundamentals of Maxwell's Kinetic Theory of a Simple Monoatomic Gas (Academic Press, New York, 1980).

34. R. S. Krupp, A nonequilibrium solution of the Fourier transformed Boltzmann equation, M.S. Thesis, MIT (1967); Investigation of solutions to the Fourier transformed Boltzmann equation, Ph.D. Thesis, MIT (1970).

35. M. H. Ernst, Phys. Reports 78, 1 (1981).

36. A. V. Bobylev, Sov. Sci. Rev. C. Math. Phys. 7, 111 (1988).

37. D. Baganoff, Phys. Fluids 14, 3403 (2002).

38. L. Acedo, A. Santos, and A. V. Bobylev, J. Stat. Phys. 109, 1027 (2002).

39. H.-D. Kim and H. Hayakawa, cond-mat/0202003 and cond-mat/0202036.

40. E. Ben-Naim and P. L. Krapivsky, Phys. Rev. E 61, R5 (2000).

41. J. A. Carrillo, C. Cercignani, and I. M. Gamba, Phys. Rev. E 62, 7700 (2000).

42. A. V. Bobylev, J. A. Carrillo, and I. M. Gamba, J. Stat. Phys. 98, 743 (2000).

43. A. Baldassarri, U. M. B. Marconi, and A. Puglisi, Europhys. Lett. 58, 14 (2002).

44. P. L. Krapivsky and E. Ben-Naim, J. Phys. A 35, L147 (2002).

45. E. Ben-Naim and P. L. Krapivsky, Phys. Rev. E 66, 011309 (2002).

46. E. Ben-Naim and P. L. Krapivsky, Eur. Phys. Jour. E 8, 507 (2002).

47. M. H. Ernst and R. Brito, Europhys. Lett. 58, 182 (2002).

48. M. H. Ernst and R. Brito, J. Stat. Phys. 109, 407 (2002).

49. M. H. Ernst and R. Brito, Phys. Rev. E 65, 040301 (2002).

50. A. V. Bobylev and C. Cercignani, J. Stat. Phys. 106, 547 (2002).

51. U. M. B. Marconi and A. Puglisi, Phys. Rev. E 65, 051305 (2002).

52. U. M. B. Marconi and A. Puglisi, Phys. Rev. E 66, 011301 (2002).

53. T. Antal, M. Droz, and A. Lipowski, Phys. Rev. E 66, 062301 (2002).

54. A. Santos, Physica A 321, 442 (2003).

55. H. Hayakawa, cond-mat/0209630.

56. V. Garzo, cond-mat/0211373.

57. A. Santos and M. H. Ernst, cond-mat/0302285.

58. P. K. Haff, J. Fluid Mech. 134, 401 (1983).

59. E. L. Grossman and B. Rooman, Phys. Fluids 8, 3218 (1996).

60. N. V. Brilliantov and T. Pöschel, Phys. Rev. E 611716 (2000).

61. H.-D. Kim and H. Hayakawa, J. Phys. Soc. Japan 70, 1954 (2001).

62. J. Lutsko, J. J. Brey, and J. W. Dufty, Phys. Rev. E 65, 051304 (2002).

63. G. E. Andrews, R. Askey, and R. Roy, Special Functions (Cambridge University Press, New York, 1999).

64. A. Rosato, K. J. Strandburg, F. Prinz, and R. H. Swendsen, Phys. Rev. Lett. 58, 1038 (1987).

65. R. Jullien, P. Meakin, and A. Pavlovitch, Phys. Rev. Lett. 69, 640 (1992).

66. J. B. Knight, H. M. Jaeger, and S. R. Nagel, Phys. Rev. Lett. 70, 3728 (1993). 
67. J. Duran and R. Jullien, Phys. Rev. Lett. 80, 3547 (1998).

68. D. C. Hong, P. V. Quinn, and S. Luding, Phys. Rev. Lett. 86, 3423 (2001).

69. M. E. Mobius, B. E. Lauderdale, S. R. Nagel, and H. M. Jaeger, Nature 414, $270(2001)$.

70. J. W. Dufty, cond-mat/0108444.

71. A. Santos and J. W. Dufty, Phys. Rev. Lett. 86, 4823 (2001).

72. A. Santos and J. W. Dufty, Phys. Rev. E 64, 051305 (2001).

73. J. C. Williams, Powder Technology 15, 245 (1976).

74. C. S. Campbell, Annu. Rev. Fluid Mech. 22, 57 (1990).

75. F. B. Pidduck, Proc. London Math. Soc. Ser. 2, 15, 89 (1915).

76. P. A. Martin and J. Piasecki, Europhys. Lett. 46, 613 (1999).

77. K. Feitosa and N. Menon, Phys. Rev. Lett. 19, 8301 (2002).

78. H. A. Lorentz, Arch. Néerl. 10, 336 (1905); for a review, see E. H. Hauge, in: Transport Phenomena, eds. G. Kirczenow and J. Marro (Springer-Verlag, Berlin, 1974).

79. R. Dickman, J. Stat. Phys. 41, 607 (1985).

80. C. Marin, V. Garzo, and A. Santos, Phys. Rev. E 51, 3812 (1995).

81. R. J. Glauber, J. Math. Phys. 4, 294 (1963).

82. E. Ben-Naim, L. Frachebourg, and P. L. Krapivsky, Phys. Rev. E 53, 3078 (1996).

83. J. S. van Zon, F. C. MacKintosh, cond-mat/0205512.

84. C. Cercignani, J. Stat. Phys. 102, 407 (2001).

85. A. Goldshtein, M. Shapiro, and C. Gutfinger, J. Fluid Mech. 316, 29 (1996).

86. V. Kamenetsky, A. Goldshtein, M. Shapiro, and D. Degani, Phys. Fluids 12, 3036 (2000).

87. A. Goldshtein, V. Kamenetsky, A. Potapov, M. Shapiro, C. Campbell, and D. Degani, Granular Matter 4, 115 (2002). 